\title{
The morphology and structure of red pigment producing Fungus: Monascus Purpureus
}

\begin{abstract}
The red pigment producing, ascospore forming fungus Monascus purpureus was obtained by monospore isolation and maintained on potato dextrose agar at $32^{\circ} \mathrm{C}$ for 7 days. $M$. purpureus produces compact colonies of mycelia and accumulates large quantities of red pigment. Here we aimed to describe this newly isolated red pigment producing fungus using biochemical and microscopy technique. A newly isolated red pigment producing fungus from local red fermented rice was identified using Microbial Identification System based on fatty acids profiles. The growth, morphology, and structure of $M$. purpureus were characterized by Scanning Electron Microscopy (SME). We found that $M$. purpureus reproduces sexually (by the formation of cleistothecium with ascospores) and asexual (by the formation of conidia). In Monascus species, the formation of either asexual or sexual spores appears to be an effective growth strategy. On the basis of biochemical and all morphological investigations it could be concluded that the new strain isolated from red fermented rice belongs to species M. purpureus, labeled as M. purpureus FTCC 5391.
\end{abstract}

Keywords: monascus purpureus, microbial identification system, scanning electron microscope (sem), morphology, asexual, sexually, ascospore, conidia
Volume 5 Issue I - 2017

\author{
Musaalbakri Abdul Manan,' Rosfarizan \\ Mohamad, ${ }^{2}$ Arbakariya Ariff² \\ 'Biotechnology and Nanotechnology Research Centre, \\ Malaysian Agricultural Research and Development Institute \\ (MARDI), Malaysia \\ ${ }^{2}$ Department of Bioprocess Technology, Universiti Putra Malaysia \\ (UPM), Malaysia
}

\begin{abstract}
Correspondence: Arbakariya Ariff, Department of Bioprocess Technology, Faculty of Biotechnology and Biomolecular Sciences, Universiti Putra Malaysia (UPM), 43400 Serdang, Selangor, Malaysia ,Tel 603-8947I055, Email arbarif@upm.edu.my
\end{abstract}

Received: May 23, 2017 | Published: May 31, 2017

\section{Introduction}

The fungus Monascuspurpureus belongs to the phylumEucomycota, subphylum Ascomycotina, class Ascomycetes, order Eurotiales, and family Monascaceae.. The genus Monascus includes nine species; M. pilosus, M. purpureus, M. ruber, ${ }^{2}$ M. floridanus. ${ }^{3}, M$. argentinensis, $M$. eremophilus, $M$. lunisporas, $M$. pallens and $M$. sanguineus. ${ }^{4,5}$ which has been internationally recognized. These fungi are a source of various polyketide-type secondary metabolites and famous for their fermented products. ${ }^{4}$ For the Japanese, the fermented products by Monascus sp. are red yeast rice, red rice, red leaven, and beni-koji, while for the Chinese it is known as hung-chu, hong qu, angkak, and zhitai. ${ }^{6}$ Monascus red rice may be marketed for these uses in the form of dried red rice or as its powder. According to Juzlova et al. ${ }^{7}, M$. purpureus is a strain of M. ruber van Tieghem, a destructive mold that grows on starch and silage and naturally exists in dairy products. The yeast causes fermentation of cellobiose, maltose, fructose, and glucose, but does not ferment cane sugar. ${ }^{8} M$. purpureus is distinguished by its usually spherical ascospores ${ }^{9}$, which are 5 microns in diameter or ovoid ( $6 \times 5$ microns). During the early stages, the young part of the M. purpureus mycelium is white. However, this rapidly changes to a rich orange and later to a distinctly rich red color, reflecting the increasing acidity of the medium and the production of red-orange hyphae. A deep crimson color is found at the substratum as the culture ages. ${ }^{6}$ Red fermented rice (also known as angkak) obtained by the cultivation of Monascus $s p$. on rice grains has been well-known as a food dye in Japan and China for centuries. ${ }^{10}$ As a result of recent efforts to replace synthetic food dyes with natural colorants, the pigments produced by Monascus $s p$. have attracted attention.

Most higher fungi exist as a heterokaryon, in which genetically dissimilar nuclei share the same mycelium. ${ }^{11} \mathrm{~A}$ fungus is homokaryotic when the colonies contain genetically identical nuclei. Heterokaryosis has been shown to cause variation in filamentous fungi and instability or loss of metabolites during fermentation processes..$^{12} \mathrm{M}$. purpureus is a member of the genus Monascus, which includes a set of fungi that are generally considered asexual, although perfect forms (forms that reproduce sexually) have also been found..$^{13}$ As is the case for many fungi, the taxonomy of $M$. purpureus is primarily based on morphological features, rather than physiological and biochemical features or genetic characteristics, as often used to classify bacteria. ${ }^{14}$

Knowledge of the structure and morphology of this fungus is important for the development of a high-performance industrial fermentation process for red pigment production. It is difficult to determine the morphology and structure for these types of strains, but not impossible. One approach is to use Scanning Electron Microscopy (SEM). However, to avoid damage to the specimen during examination in the SEM chamber, a suitable preparation technique must first be developed. If the cells can be kept hydrated, mechanical disruption of the cells can be minimized, allowing clearer determination of cell morphology and structure. Here we use SEM together with an improved sample preparation method to determine the morphology and structure of M. purpureus, which was recently isolated from local red fermented rice. Later, the isolated strain identified using Microbial Identification System (MIS) by gas chromatography analysis of fatty acid methyl esters (GC-FAME). The result obtained was used to summarize their unique fatty acid profiles and the present state of structure and morphology of this fungus.

\section{Materials and methods}

\section{Microorganism and maintenance}

The red pigment producing fungus was isolated from local red fermented rice, and the culture was preserved at the Collection of Functional Food Culture (CFFC), Biotechnology and Nanotechnology Research Centre, Malaysian Agricultural Research and Development Institute (MARDI), Malaysia. The strain's ability to produce red pigment was improved using the monospore isolation method. The monospore isolation of the red pigment producing strain was carried out by repeated sub culturing on solid enrichment medium using the spread plate technique and incubation at $32^{\circ} \mathrm{C}$ for 7 days to obtain progeny at different stages of the subculture. The obtained progeny 
were subcultured on solid enrichment medium, and the single colonies or monospores that were obtained were then transferred to slants for spore formation. The red pigment production of each single colony was compared, and the best red pigment producer was selected for further study. A stock culture of the red pigment producer in the form of ascospores, which were attached to porous ceramic beads, was suspended in cryopreservative. The culture was placed in a vial and freeze-dried using a laboratory freeze dryer according to the standard method. The freeze-dried stock culture was stored in a vial at $-80^{\circ} \mathrm{C}$ prior to use in spore production (Methods by Technical Service Consultants Limited, Europe's Leading Manufacturer of Microbiological Consumables; Mixed Vials of Protected in Polystyrene Tray, Cat. No.: TS70-AS). ${ }^{15}$ The stock culture was aseptically subcultured on potato dextrose agar (PDA) for 7 days at $32^{\circ} \mathrm{C}$ for subsequent use.

\section{Strain identification}

The strain was identified using the MIS (Standard Microbial Identification System Operating Manual, 2001) for fungus based on cell fatty acid profiles. This method of identification is not limited to any specific microbial grouping, but it can be used to identify specific microorganism isolated as pure culture in-vitro. ${ }^{16,17}$ The method involves several steps, which includes the standard culture technique, cell harvesting, saponification, methylation and extraction of fatty acid methyl esters. The profiles of fatty acids were then determined using Gas Chromatography (HP, Agilent Technologies Inc. USA) equipped with a flame ionization detector (FID). The column used was HP-5, 30m x $0.32 \mathrm{~mm} \times 0.25 \mathrm{~mm}$ (cross linked 5\% Ph Me Silicon, Agilent Technologies Inc. USA). The flow rate of carrier gas (N2) was set at $40 \mathrm{~mL} / \mathrm{min}$ and the temperatures of the injector port, column and detector were set at $250 \mathrm{oC}, 270 \mathrm{oC}$ and $300 \mathrm{oC}$, respectively. The volume of sample injected was $1 \mathrm{~mL}$. Every signal peak produced from the Gas Chromatography detector is then stored on the hard disk and the fatty acid methyl ester composition of the sample is compared to a stored database using the Sherlock pattern recognition software. ${ }^{18}$

\section{Examination of structure and morphology}

A scanning electron microscope (Philips XL30, Netherlands) was used to determine the structure and morphology newly-isolated of M. purpureus. The SEM samples were prepared as follows. After culturing on potato dextrose agar (PDA) for 7 days at $32^{\circ} \mathrm{C}$ (until the appearance of red pigment), the cells were fixed in glutaraldehyde fixative $(2.5 \%$.w/v glutaraldehyde in buffer solution, $\mathrm{pH} 7.2)$ overnight at $4{ }^{\circ} \mathrm{C}$. The cells were then removed from the glutaraldehyde fixative and washed three times with $0.1 \mathrm{M}$ sodium cocodylate buffer $(\mathrm{pH} 7.0)$ for $15 \mathrm{~min}$. After rinsing, the clean cells were then fixed in $1 \%(\mathrm{w} / \mathrm{v})$ osmium tetroxide $(\mathrm{OsO} 4)$ at $4^{\circ} \mathrm{C}$ for $4 \mathrm{~h}$. The cells were then removed from the $\mathrm{OsO} 4$ solution and dried using serially diluted high-grade ethanol $(10 \%, 20 \%, 30 \%, 40 \%, 50 \%, 60 \%, 70 \%, 80 \%, 90 \%$, and $100 \% . v / v)$ for 15 min each. The $80 \%, 90 \%$ and $100 \%$ ethanol steps were done twice each. Acetone was used to dehydrate the cells prior to critical point drying using compressed carbon dioxide transition fluid (Baltec $030 \mathrm{CPD}$, Switzerland). The cells were then mounted onto SEM specimen stubs with the ectodermal layers facing upwards and sputter coated (Polaran E5100 SEM Coating Unit, United Kingdom) with gold. The SEM was operated at $15 \mathrm{kV}$ and high vacuum pressure.

\section{Results and discussion}

\section{Strain identification using MIS method}

The peak time, width, height and area data of the analysis are transmitted from the integrator to the computer at the end of each run where the peaks are named, the library is searched and report is printed. Each peak from the chromatographic analysis is listed by retention time (RT), area and area/height ratio (RT/HT). Included in the composition report is the equivalent chain length (ECL), a linear interpolation peak's retention between two saturated straight-chain fatty acid (methyl ester) reference peaks. The Microbial Identification System (MIS) software compares the ECL of each peak in the analysis with the expected ECL of the fatty acids in the peak naming table. The accuracy of the system was monitored by injecting the sample extract prepared from known fungus of $M$. purpureus (England). The result shows that the known fungus was matched and confirmed as M. purpureus at the similarity index of 0.597 (Table 1). A total of four 4 different cultures isolated from local red fermented rice were identified. Table 2 shows a typical report of library search of one of the isolates. The result indicates that the similarity index of unknown sample of fungus extract is 0.708 that matched to M. purpureus (Table 1). The highest similarity index determines the most likely matches. Therefore, the culture of the unknown sample belongs to the species of $M$. purpureus and namely as M. purpureus FTCC 5391.

Table I Report of known fungus M. purpureus (England)

\begin{tabular}{ll}
\hline Solvent Area & $\mathbf{3 9 4 3 6 0 0 0}$ \\
\hline Total area & $2168 \mathrm{I}$ \\
Name area & $2168 \mathrm{I}$ \\
\% Name & 100 \\
Total amount & 19754 \\
ECL deviation & 0.001 \\
Ref ECL shift & 0.002 \\
\hline
\end{tabular}

TSBA (rev 4.0) Monascus : 0.597 M. purpureus : 0.597

Table 2 Library search report of unknown sample

\begin{tabular}{ll} 
Solvent Area & 3741000 \\
\hline Total area & 266181 \\
Name area & 261183 \\
\% Name & 98 \\
Total amount & 247700 \\
ECL deviation & 0.005 \\
Ref ECL shift & 0.014 \\
\hline
\end{tabular}

TSBA (rev 4.0) Monascus : 0.708; M. purpureus : 0.708

\section{Structures and growth morphology}

When a single spore of $M$. purpureus FTCC 5391was cultivated on PDA, it germinated with slender hyphae or a germ-tube emerging from the spore. Hyphal growth is apical, with wall extension being limited to the roughly hemispherical apex of the hyphae. ${ }^{19}$ During growth, nutrients were absorbed from the substratum; nuclear division and hyphal branching occurred to give an approximately circular colony, in which the diameter increased at a uniform rate (data not shown). The size and shape of the spores and colonies of filamentous fungi are important factors for fungal identification. The hyphae of a growing colony are termed mycelium. ${ }^{20}$ A single colony of $M$. purpureus FTCC 5391 grown on PDA after 7 days at $32^{\circ} \mathrm{C}$ is shown in Figure 1.

The colony of $M$. purpureus FTCC 5391 was thin and spreading with reddish in color, indicating that a substantial amount of red pigment had been produced during growth.

Figure 2 shows SEM micrographs of the mycelium of $M$. purpureus FTCC 5391 that was not treated prior to SEM. From these 
micrographs, the morphology and growth of the fungus cannot be clearly identified, and some cell damage can be seen.

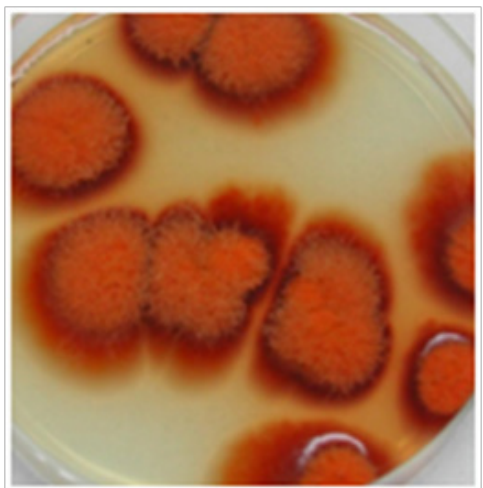

Figure I Monascus purpureus FTCC 539 I growing on PDA after 7 days of growth at $32^{\circ} \mathrm{C}$. The last image was taken from a single spore through monospore isolation method.

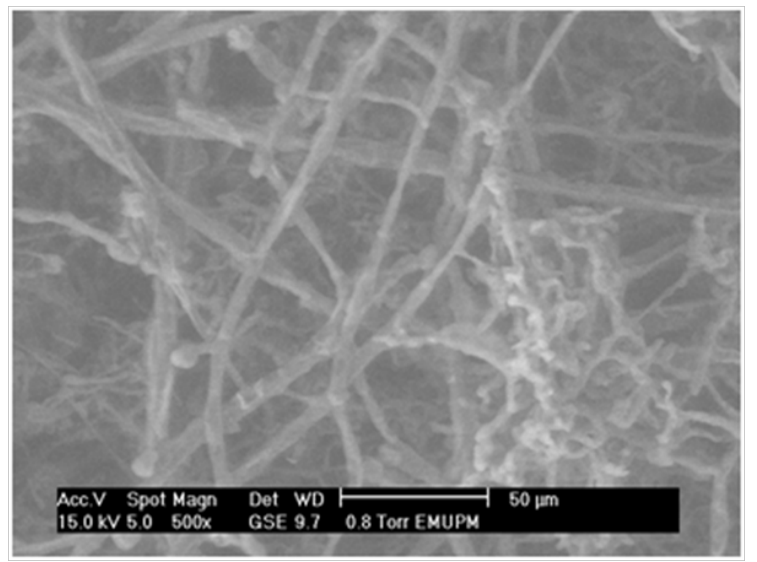

Figure 2 Scanning electron micrograph of untreated Monascus purpureus FTCC 539I. Magnification $=500 \times$ and I,000x; scale bar $=50 \mu \mathrm{m}$ and $20 \mu \mathrm{m}$, respectively.

Figure 3 shows SEM micrographs of M. purpureus FTCC 5391 mycelia that had been pretreated prior to SEM. The mycelia were numerous and round, including both thin-walled ascocarps and chains of conidia. It can also be seen that the mycelia extended through the capillaries of the agar. The exogenous conidia formed during the growth of M. purpureus FTCC 5391 were successively separated as chains (Figure 4).

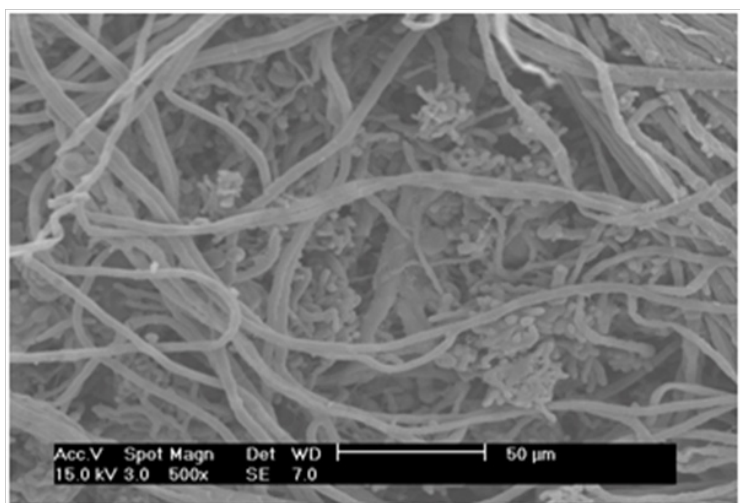

Figure 3 Scanning electron micrograph of $M$. purpureus FTCC 539 mycelium. Magnification $=200 \times, 500 \times, 800 \times$; scale bar $=100,5020,20 \mu \mathrm{m}$, respectively .

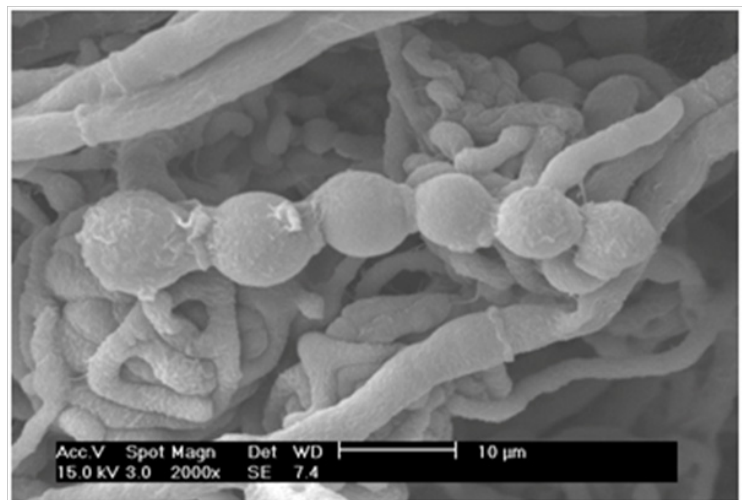

Figure 4 Scanning electron micrograph of conidia of $M$. purpureus FTCC 539l. Magnification $=800 \times, 2,000 \times, 4,000 \times$, scale bar $=20,10,5 \mu \mathrm{m}$, respectively.

We found that $M$. purpureus FTCC 5391 produces its asci in complex fruiting bodies, known as ascocarps (Figure 5). From the micrograph shown in Figure 5, it can be seen that the asci of $M$. purpureus FTCC 5391 are present singly and grouped within ascocarps, which are formed by branching and intertwining adjacent hyphae. Examination of the stalk, cap, and gills shows that the fruit bodies are composed of long, cylindrical branching threads, known as hyphae. The hyphae are divided by cross-walls into compartments that typically contain several nuclei. The role of the edible fruit bodies (or fruiting bodies) is to produce large numbers of spores, by which dispersal occurs..$^{20}$ The fruiting body is a special feature of cultivated fungi, and its formation is dependent on nutrition and the ability of an extensive mycelium able to penetrate the solid media. ${ }^{20}$

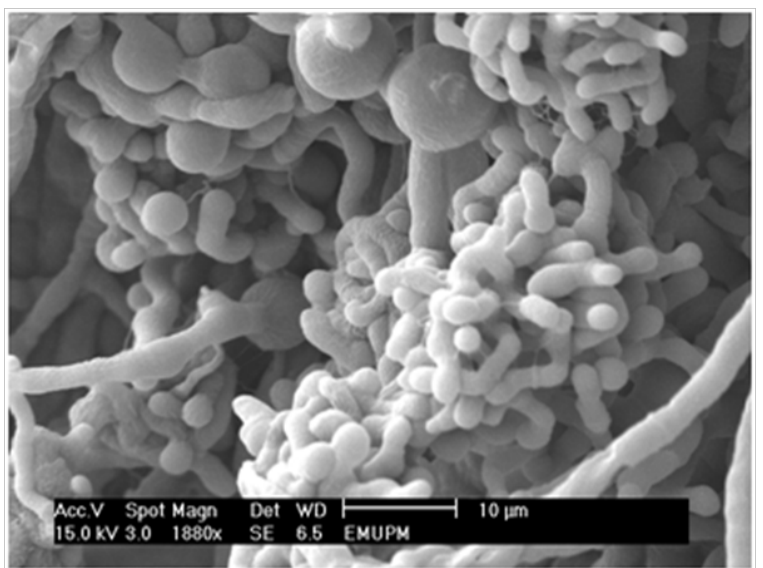

Figure 5 Scanning electron micrograph of young ascocarps of $M$ purpureus FTCC 5391. Magnification $=1880 \times$, scale bar $=10 \mu \mathrm{m}$.

Like many other fungi, the sexual reproduction stage of $M$. purpureus FTCC 5391 is initiated by the formation of cleistothecium (Figure 6). From the micrograph, it can be seen that cleistothecium consists of a group of asci and ascospores within a spherical, smoothwalled body. The cleistothecium is formed by special copulation branches. Such a growth morphology is generally considered advantageous for industrial fungi used in fermentation processes. ${ }^{21}$ Members of the phylum Eucomycota, commonly referred to as the Ascomycetes, are those fungi in which the sexual process involves the production of haploid ascospores through the meiosis of a diploid nucleus in an ascus. ${ }^{22}$. Most Ascomycetes also perform asexual sporulation, conidiospores (conidia) being produced on specialized aerial hyphae that that rise above the substratum. ${ }^{23}$ Suh \& $\operatorname{Shin}^{13}$ 
proposed that asexual sporulation is a common reproductive mode for Monascus species. As part of this, the hyphae form an interwoven mat called a mycelium, which acts as the feeding network of a fungus. ${ }^{24}$

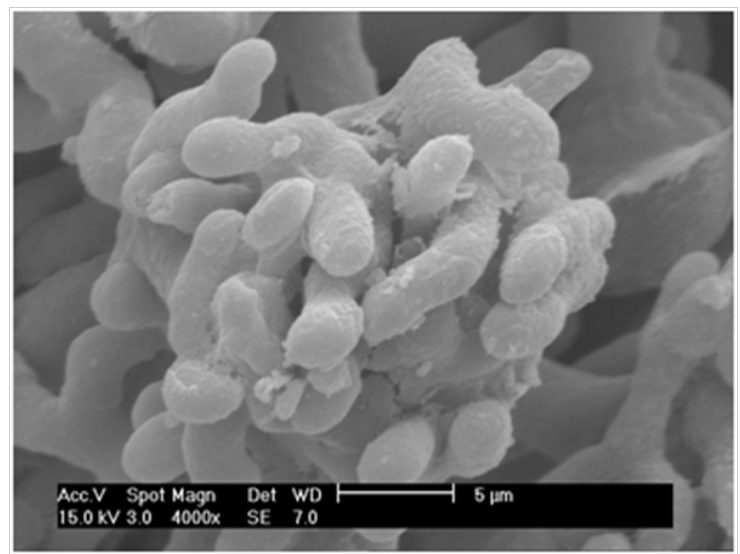

Figure 6 Scanning electron micrograph of cleistothecium. Magnification = $500 \times$ and 4,000x; scale bar $=50$ and $5 \mu \mathrm{m}$, respectively.

M. purpureus FTCC 5391 is typically well-developed, branched and septate, saprophytic, forming conidia and sporangium-like asci (ascocarps and cleistothecium, terminal with and individual hyphal walls), with ascithat areenclosed by interwoven hyphae. Here we found that M. purpureus FTCC 5391 can reproduce sexually (involving the formation of cleistothecium with ascospores) and asexually (by the formation of conidia), which strictly aerobic as described by. ${ }^{19}$ Under favorable environmental conditions, each conidium can produce a young mycelium..$^{25}$. Using yeast extract to stimulate conidiation can repress the sexual cycle of M. purpureus FTCC 5391 and increase biomass production. ${ }^{7}$. Sodium nitrate increases the rate of sporulation in M. purpureus FTCC 5391, but limits growth and results in intermediate pigment yields. Whereas addition of ammonium chloride represses conidiation and the sexual cycle and produces high pigment yields. ${ }^{26}$ The use of polysaccharides, disaccharides, and monosaccharides as carbon sources, together with nitrogen, have been implicated in the onset of radial growth and conidiation, as well as with enhanced cell production and variation in colony morphology. ${ }^{23}$ According to lizuka \&Lin, ${ }^{19}$ the sexual phase of an M. purpureus is now termed the teleomorph, and the asexual phase the anamorph; the concept of "perfect" and "imperfect" states have been abandoned. Isolates are often obtained that produce a telemorph, but from their asexual sporulation are clearly identical with known Ascomycetes ${ }^{27}$. When both anamorphic and teleomorphic phases are present, the teleomorph name is used. ${ }^{19}$.

Bacteria, yeast, and fungi have been used in industria biofermentation for the production of various useful products. ${ }^{28}$ Fungi are more evolutionarily advanced than prokaryotes (e.g., prions, viruses, and bacteria). ${ }^{11}$ Fungi are classified as eukaryotes that have a diploid number of chromosomes, a nuclear membrane, and have sterols in their plasma membrane. Most fungi occur in the hyphae form as branching, thread-like tubular filamentous structures, either with (septate) or without (coenocytic) cross walls. ${ }^{22}$ A mass of hyphal elements is termed the mycelium. Aerial hyphae often produce asexual reproduction propagules termed conidia (synonymous with spores). ${ }^{29}$

In fungal biofermentation, either spore suspensions or vegetative cells can be used as the inoculum. In cases where vegetative cells must be used as the inoculum, it would be advantageous to develop spore suspension inoculums. This is because spores are easier to handle, are more viable, and can be stored for longer. The sporulation rate of Monascus species is low because of its asci feature. Therefore, vegetative cells are widely used as the inoculum in fermentation processes involving Monascus species. ${ }^{30}$ The sporulation rate of Monascus species can be improved by optimizing the composition of the growth medium. ${ }^{23,31}$ Fungi sporulation is related to a sophisticated system of positive and negative gene regulations that are affected by environmental and nutritional factors. ${ }^{32}$ Temporal and spatial regulation of gene expression, cell specialization, and intercellular communication might affect conidiation in fungal systems. ${ }^{25,29}$ Manipulation of the medium composition is the simplest and most effective method for improving fungi cultivation.

\section{Conclusion}

Monascus species produce naturally fermented pigments that have many advantages. These pigments can be used as coloring agents, as nutraceuticals, and in medicine. Monacolin $\mathrm{K}$ (anti-hypercholesterolemic) is the major secondary metabolite of Monascus species. Here, SEM was identified as a promising method for visualizing the morphology and structure of Monascus fungi. By SEM, following a pretreatment protocol, we were able to characterize the growth morphology and structure of M. purpureus FTCC 5391. We found that M. purpureus FTCC 5391 can reproduce sexually and asexually. Such findings are likely to be important for successful cultivation of $M$. purpureus FTCC 5391 and similar fungi, as well as for the optimization of industrial fungal fermentation processes. The red-pigment producing fungus isolated locally from red fermented rice was identified MIS based on fatty acids profiles as M. purpureus and namely as FTCC 5391. Further studies should now address the relationship between the genetic mechanisms controlling sporulation and nutritional necessity.

\section{Acknowledgements}

None.

\section{Conflicts of interest}

None.

\section{Funding}

None.

\section{References}

1. Aniya Y, Yokomakura T, Yonamine M, et al. Protective effect of the mold Monascus anka against acetaminophen-induced liver toxicity in rats. Jpn J Pharmacol. 1998;78(1):79-82.

2. Hawksworth DL, Pitt JI. A new taxonomy for Monascus species based on cultural and microscopical characters. Aust J Bot. 1983;31(1):51-61.

3. Barnard EL. A new species of Monascus from pine tissues in Florida. Mycologia. 1987;79(3):479-484.

4. Chen W, Zhou Y, Shao Y, et al. Edible filamentous fungi from the species Monascus: Early traditional fermentations, moderns molecular biology, and future genomics. Comprehensive Reviews in Food Science and Food Safety. 2015;14(5):555-567.

5. Shao Y, Lei M, Mao Z, et al. Insights into Monascus biology at the genetic level. Appl Microbiol Biotechnol. 2014;98(9):3911-3922.

6. Li C, Jiang Q, Li Y. Monascus purpureus-fermented rice (red yeast rice): a natural food product that lowers blood cholesterol in animal models of hypercholesterolemia. Nutrition Research. 1998;18(1):71-81. 
7. Juzlova P, Martinkova L, Kren V. Secondary metabolites of the fungus Monascus: A review. Journal of Industrial Microbiology. 1996;16(1):163-170.

8. Lin TF, Yakushijin $\mathrm{K}$, Buchi $\mathrm{GH}$, et al. Formation of watersoluble Monascus red pigments by biological and semi-synthetic processes. Journal of Industrial Microbiology. 1992;9(3):173-179.

9. Rasheva T, Hallet JN, Kujumdzieva A. Taxonomic investigation of Monascus purpureus 94-25 strain. Journal of Culture Collections. 1998;2(1):51-59.

10. Journoud M, Jones JH. Red yeast rice: A new hypolipidemic drug. Life Sci. 2004;74(22):2675-2683.

11. Carlile MJ, Watkinson SC. The fungi. (2nd edn) Academic Press, San Diego, California, USA. 1994:588.

12. Kale S, Bennet JW. Strain Instability in filamentous Fungi. In: Bhatnagar D \& Lillehoj EB (Eds.) Handbook of Applied Mycology: Mycotoxin in Ecology System. (5th edn), New York, USA. 1992:311-333.

13. Suh JH, Shin CS. Analysis of the morphologies changes of Monascus sp. J101 cells cocultured with Saccharomyces cerevisiae. FEMS Microbiology Letters. 2000;193(1):143-147.

14. Shepherd D, Carels M. Product formation and differentiation in fungi. In: Smith JE (Eds.) Fungal Differentiation, A Contemporary Synthesis (4th edn) Mycology, Marcell Dekker, New York, USA. 1983:515-535.

15. Musaalbakri AM, Samat N, Kasran M, et al. Proximate and amino acids composition of Monascus fermented products with potential as functional feed ingredients. Cogent Food and Agriculture. 2017;3(1):1295767.

16. Moss CW. Gas-liquid chromatography as an analytical tool in microbiology. J Chromatogr. 1981;203:334-347.

17. Moss CW, Shinoda T, Samuels JW. Determination of cellular fatty acid compositions of various yeasts by gas-liquid chromatography. $\mathrm{J}$ Clin Microbiol. 1982;16(6):1073-1079.

18. Sasser M. Bacterial identification by gas chromatographic analysis of fatty acid methyl esters (GC-FAME). Technical note 101, MIDI. 2006:6.

19. Iizuka H, Lin CF. On the genus Monascus of Asia and its specific characteristics. Advances in Biotechnology. 1980;2:555-561.

20. Tortora GJ, Funke BR, Case CL. Mirobiology: An introduction. (5th edn), The Benjamin/Cummings Publishing Company, USA. 1994:295303 .
21. Pitt JI, Hocking AD. Fungi and food spoilage. Food Science and Technology; A Series of Monographs. Academic Press Australia. India. 1985:121-123.

22. Carel M, Shepherd D. Sexual reproduction cycle of Monascus in submerged shaken culture. Journal of Bacteriology. 1975;122(1):288294

23. Ajdari Z, Ebrahimpour A, Musaalbakri AM, et al. Nutritional requirements for the improvement of growth and sporulation of several strains of Monascus purpureus on solid state cultivation. Journal of Biomedicine and Biotechnology. 2011:9.

24. Campbell NA. Biology. (4th edn), The Benjamin/Cummings Publishing Company, New York, USA. 1995:573-585.

25. Krasniewski I, Molimard P, Feron G. Impact of solid medium composition on the conidition in Penicillium camemberti. Process Biochemistry. 2006;41(6):1318-1324.

26. Mostafa ME, Abbady MS. Secondary metabolites and bioactivity of the Monascus pigments review article. Global Journal of Biotechnology and Biochemistry. 2014;9(1):1-13.

27. Park HS, Yu JH. Genetic control of asexual sporulation in filamentous fungi. Curr Opin Microbiol. 2012;15(6):669-677.

28. Xie L, Chen H, Yang J. Conidia production by Beauveria bassiana on rice in solid-state fermentation using tray bioreactor. Advanced Materials Research. 2013;610-613:3478-3482.

29. Adams TH, Wieser JK, Yu JH. Asexsual sporulation in Aspergillus nidulans. Microbiol Mol Biol Rev. 1998;62(1):35-54.

30. Shin CS, Kim HJ, Kim MJ, et al. Morphological change and enhanced pigment production of Monascus when cocultured with Saccharomyces cerevisiae or Aspergillus oryzae. Biotechnol Bioeng. 1998;59(5):576581

31. Gao L, Sun MH, Liu XZ, et al. Effects of carbon concentration to nitrogen ratio on the growth and sporulation of several biocontrol fungi. Mycol Res. 2007;111(1):87-92.

32. Jackson MA, Schisler DA. The composition and attibutes of Colletotrichum truncatum spores are altered by nutritional environment. Appl Environ Microbiol. 1992;58(7):2260-2265. 